\title{
Contact problems for particles in a shear flow
}

\author{
Nicolas Verdon* - Aline Lefebvre-Lepot** — Laurent Lobry*** \\ Patrice Laure*
}

* Laboratoire J.-A. Dieudonné, CNRS UMR 6621

Université de Nice - Sophia Antipolis, Parc Valrose

F-06108 Nice cedex 02

\{verdon.nicolas; patrice.laure\}@unice.fr

** Centre de Mathématiques Appliquées, Ecole Polytechnique route de Saclay, F-91128 Palaiseau cedex

lefebvre@cmap.polytechnique.fr

*** Laboratoire de Physique de la Matière Condensée, CNRS UMR 6622

Université de Nice-Sophia Antipolis, Parc Valrose

F-06108 Nice cedex 2

laurent.lobry@unice.fr

ABSTRACT. This paper focuses on improving the description of the contact between solid particles in a fluid flow. The numerical approach used is related to the fictitious domain method for the fluid-solid problem. It is associated to a gluey particle model in order to improve the behaviour of the particles during their contacts as a Lagrangian method is applied for their displacement. The numerical methodology is validated through $2 D$ and $3 D$ computations describing interactions of two particles in a shear flow. The results obtained show the ability of the scheme to recover the reversibility of the Stokes equations, even for $3 D$ configurations. Finally, another example is studied with larger number of particles.

RÉSUMÉ. On s'intéresse à l'amélioration de la description du contact entre plusieurs particules solides dans un écoulement fluide. Le modèle numérique est la méthode des domaines fictifs pour la description du problème fluide-solide. Elle est associée à un modèle de contact visqueux qui intervient lors du déplacement Lagrangien des particules. Le schéma numérique a été validé sur des calculs $2 D$ et $3 D$ décrivant l'évolution de deux particules dans un écoulement cisaillé. Les résultats obtenus montrent la capacité du schéma à retrouver la réversibilité des équations de Stokes tout en évitant le recouvrement même pour des configurations 3D. Enfin, un exemple avec plus de particules est présenté.

KEYWORDS: suspension, contact, fictitious domain method, level-set, gluey model, Stokes equation.

MOTS-CLÉS : suspension, contact, méthode des domaines fictifs, level-set, modèle de contact visqueux, équation de Stokes.

DOI:10.3166/EJCM.19.513-531 C 2010 Lavoisier, Paris

EJCM - 19/2010. Fluid-structure interaction, pages 513 to 531 


\section{Introduction}

Since the emergence of the fictitious domain methods in the 2000's (Glowinski et al., 1999; Singh et al., 2000; Patankar et al., 2000), lots of multiphase applications have been treated that way, see for instance (Glowinski et al., 2001; Wachs, 2009; Coupez et al., 2009). The advantages of these methods are straightforward: fluid/solid or multiphase problems can be treated with an Eulerian approach on the whole domain because the rigidity condition can be added in the weak formulation.

In this work, we study the displacement of solid particles in a shear flow. For this purpose, a characteristic function is used to describe the particle domain and therefore allows to extend all variables associated to each phase to whole computational domain thanks to mixing relations. Then the displacement of the particles and the corresponding characteristic function is achieved by using a Lagrangian approach.

As the distance between particles can be very small (Meunier et al., 2008), typically around $10^{-5}$ times the characteristic size of particles. Another important point is to deal with particles overlapping. This will be achieved by introducing contact models, especially the gluey particle model (Maury, 2007; Lefebvre, 2009) which takes into account viscous effects between particles.

\section{Numerical Modelling}

This work is carried out using an immersed domain approach using a level set function for determining the interface between fluid and solids (Coupez et al., 2009). The weak formulation of the mixed fluid-solid system is written by mean of a Lagrange multiplier. At last, the displacement of the solid particles is carried out with a Lagrangian approach, which includes the gluey model. These points are described hereafter.

\subsection{The immersed domain approach}

The immersed domain method is achieved by splitting the computational domain $\Omega$ into two subdomains $\Omega_{f}$ and $\Omega_{s}$ for respectively the fluid and solid parts (see Figure 1). In the case of multiple particles, the solid domain is the union of domain corresponding to each particle, namely $\Omega_{s}=\bigcup_{i}^{N} \Omega_{s_{i}}$ for $N$ particles.

The interface $\Gamma_{s}$ between the two phases is described by the zero isosurface of a distance function:

$$
\alpha(\mathbf{x}, t)=\left\{\begin{array}{l}
>0 \text { if } \mathbf{x} \in \Omega_{s} \\
<0 \text { if } \mathbf{x} \notin \Omega_{s}
\end{array}\right.
$$




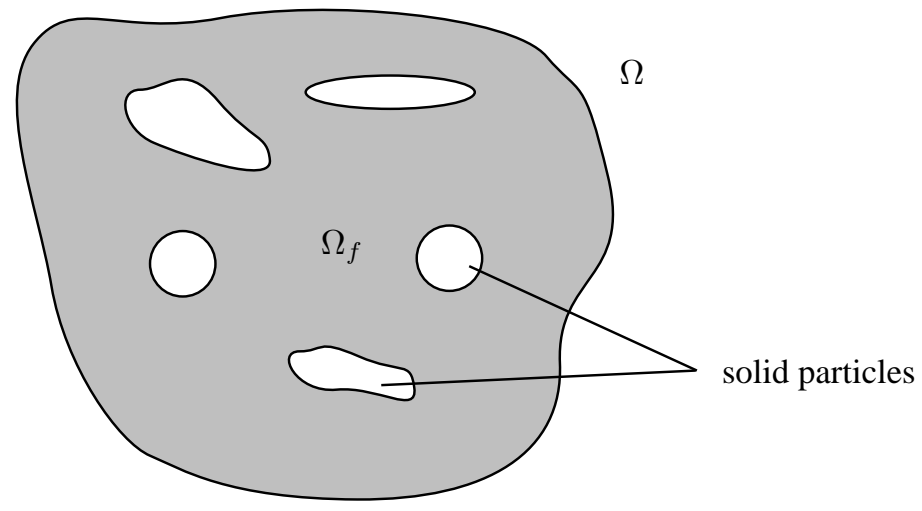

Figure 1. Schematic representation of computational domain

In practice, for a spherical particle $i$ of center $\mathbf{X}_{i}$ and radii $a_{i}$, the level set function is defined from the following signed distance:

$$
\alpha_{i}(\mathbf{x})=a_{i}-\left\|\mathbf{X}_{i} \mathbf{x}\right\|
$$

Obviously, the signed distance $\alpha$ for $N$ spherical particles can be written as:

$$
\alpha(\mathbf{x})=\max _{i=1, \cdots, N} \alpha_{i}(\mathbf{x})
$$

Finally, a "smooth" characteristic function is deduced from the level set function by taking

$$
\mathbb{I}(x, t)=\left\{\begin{array}{l}
=1 \text { if } \alpha(x)>e \\
=\frac{\alpha}{e} \text { if } 0<\alpha(x)<e \\
=0 \text { if } \alpha<0
\end{array}\right.
$$

where $e$ the mixing thickness depends on the mesh size around the interface. Note that the mixing area is inside the solid domain. In addition, the viscosity $\eta$ will be defined thanks to mixing relation:

$$
\eta=\mathbb{I} \eta_{s}+(\mathbb{E}-\mathbb{I}) \eta_{f}
$$

where $\eta_{f}$ is the fluid viscosity and $\eta_{s} \simeq r \eta_{f}$ the solid viscosity (or penalisation factor) usually taken much bigger than $\eta_{f}\left(r \approx 10^{3}\right)$. 


\subsection{Governing set of equations and weak formulation}

Neglecting inertia and gravity, the fluid-solid problem can be written with the following set of equations:

$$
\begin{cases}\nabla \cdot \sigma & =0 \\ \nabla \cdot \mathbf{u} & =0 \\ \sigma & =2 \eta_{f} \dot{\epsilon}(\mathbf{u})-p \mathbf{E} \\ {[[\mathbf{u}]]_{\Gamma_{s}}} & =0 \\ {[[\sigma \cdot \mathbf{n}]]_{\Gamma_{s}}} & =0 \\ \mathbf{u} & =\mathbf{u}_{\Gamma} \text { on the external boundary } \Gamma\end{cases}
$$

where $\mathbf{u}$ is the fluid velocity, $\dot{\epsilon}(\mathbf{v})$ the rate of strain tensor, $\sigma$ the stress tensor, $p$ the pressure, $\eta_{f}$ the fluid viscosity (the symbol $[[f]]_{\Gamma_{s}}$ means the jump of scalar $f$ across the interface $\Gamma_{s}$ ). Patankar et al. (Patankar et al., 2000) have proposed to extend the above Stokes equation to the solid domain thanks to a Lagrange multiplier by using the rigidity condition $\dot{\epsilon}(\mathbf{v})=0$ on $\Omega_{s}$. In this way, the motion in solid domain $\Omega_{s}$ corresponds to a fluid motion with an additional stress field. This is equivalent to take the stress tensor $\sigma$ inside the solid domain of the form

$$
\sigma=2 \eta_{s} \dot{\epsilon}(\mathbf{u})-p \mathbb{E}+\dot{\epsilon}(\lambda)
$$

Due to the rigid motion constraint the two first terms are zero; $\eta_{s}$ can play the role of a penalization factor of the constraint $\dot{\epsilon}(\mathbf{u})=0$, and the symmetrical tensor $\dot{\epsilon}(\lambda)$ is the Lagrange multiplier associated to this constraint.

If we consider Dirichlet boundary conditions, the weak formulation of the fluidsolid problem can be written as follows: find $(\mathbf{u}, p, \lambda)$ such that $\forall(\mathbf{v}, q, \mu) \in$ $\mathcal{H}^{1}(\Omega) \times \mathcal{L}_{0}^{2}(\Omega) \times \mathcal{H}^{1}\left(\Omega_{s}\right):$

$$
\left\{\begin{aligned}
0 & =\int_{\Omega} 2 \eta \dot{\epsilon}(\mathbf{u}): \dot{\epsilon}(\mathbf{v}) d \Omega-\int_{\Omega} p \nabla \cdot \mathbf{v} d \Omega+\int_{\Omega_{s}} \dot{\epsilon}(\lambda): \dot{\epsilon}(\mathbf{v}) d \Omega \\
0 & =\int_{\Omega} q \nabla \cdot \mathbf{u} d \Omega \\
0 & =\int_{\Omega_{s}} \dot{\epsilon}(\mu): \dot{\epsilon}(\mathbf{v}) d \Omega
\end{aligned}\right.
$$

where $\mathcal{H}^{1}(\Omega)$ and $\mathcal{L}_{0}^{2}(\Omega)$ are respectively the Sobolev and Lebesgue spaces.

The system of Equations [8] is solved using the augmented Lagrangian method and an Uzawa algorithm (Fortin et al., 1983) where the field $\lambda$ has been extended to the whole domain $\Omega$ by using the characteristic function. 
Hence,the weak formulation of the problem reads, at step $k$ of the Uzawa procedure (Laure et al., 2007):

$$
\begin{aligned}
\int_{\Omega} 2 \eta \dot{\epsilon}\left(\mathbf{u}^{k}\right): \dot{\epsilon}(\mathbf{v}) d \Omega- & \int_{\Omega} p^{k} \nabla \cdot \mathbf{v} d \Omega \\
\int_{\Omega} q \nabla \cdot \mathbf{u}^{k} d \Omega & =0
\end{aligned}
$$

whereas the update of Lagrangian multiplier reads

$$
\lambda^{k}=\lambda^{k-1}+\mathbf{u}^{k}
$$

\subsection{Particle displacements}

Solving Equations [8] gives the velocity field that allows us to compute the new particle positions (at time $t^{n+1}=t^{n}+\Delta t$ ) with a Lagrangian approach. Many different choices are possible ; the most straightforward is the Euler explicit scheme:

$$
\mathbf{X}_{i}\left(t^{n+1}\right)=\mathbf{X}_{i}\left(t^{n}\right)+\Delta t \mathbf{u}\left(\mathbf{X}_{i}\left(t^{n}\right), t^{n}\right)
$$

But this first order scheme can lead to inaccurate displacements especially for rotating velocity fields, that is why the second order Adams-Bashforth scheme is usually preferred:

$$
\mathbf{X}_{i}\left(t^{n+1}\right)=\mathbf{X}_{i}\left(t^{n}\right)+\frac{\Delta t}{2}\left[3 \mathbf{u}\left(\mathbf{X}_{i}\left(t^{n}\right), t^{n}\right)-\mathbf{u}\left(\mathbf{X}_{i}\left(t^{n-1}\right), t^{n-1}\right)\right]
$$

\subsection{Overlapping of particles}

In some computational conditions, even for non-dense suspensions, a non-physical overlapping can occur between two particles (see Figure 2). This is most often due to a large time step but it can even occur for a good set of computational parameters. Typically, that can be observed when the distances between particles becomes close to the computer accuracy. In the literature it has been observed that the minimum distance between two particles can be very small, especially for 3D spheres in a shear flow (Meunier et al., 2008). These configurations can lead to overlapping in our numerical procedure, that is why it is crucial to improve the treatment of the contact between particles.

In previous works, different approaches have been used to prevent the overlapping:

- using a simplified version of the lubrication force (linear) in the weak formulation of the problem in order to recover the same effects. In fact using directly the lubrication force would lead to non-linearities that are really difficult and computationally expensive to solve, 


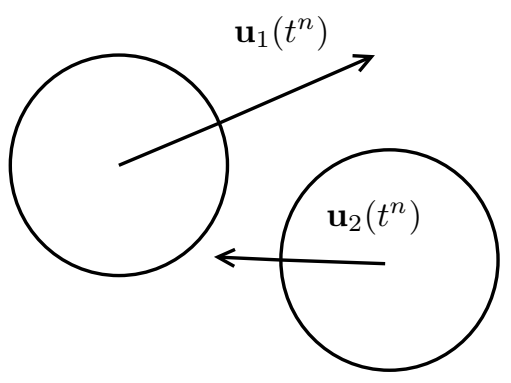

(a) at time $t^{n}$

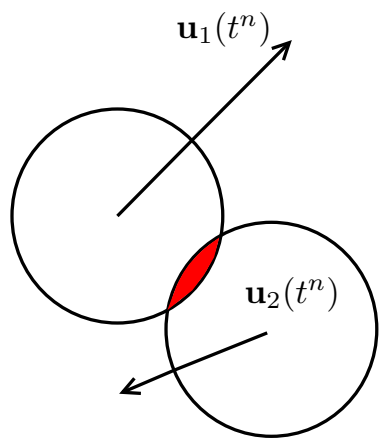

(b) at time $t^{n+1}$ after particle displacements

Figure 2. Overlapping between two particles

- using a velocity correction. Typically, it consists in moving the particle towards the normal between two particles if the distance between them becomes too small with respect to accuracy of numerical method. This correction has been used in this study and it is explained in the sequel.

\subsection{The gluey model}

As explained in the past section, the correction strategies that have been used in previous works are not rigorous enough to avoid the overlapping phenomenon. In order to improve the performance of our numerical scheme, especially for recovering the reversibility of the 3D Stokes flow, we implemented a contact model during the particle displacement with Adams-Bashforth scheme. This model, originally developed in (Maury, 2007) for the contact between two particles, has been generalized by Lefebvre for dry granular flows (Lefebvre, 2009).

\subsubsection{Principles of gluey model}

Once again, the principle of this method is based on lubrication force as shown in Figure 3. The hydrodynamic lubrication force for spherical particle is recalled hereafter:

$$
\mathbf{F}_{i j}=6 \pi a^{2} \eta_{f} \frac{\left(\mathbf{u}_{j}-\mathbf{u}_{i}\right) \cdot \mathbf{e}_{i j}}{\left\|\mathbf{X}_{i} \mathbf{X}_{j}\right\|-\left(a_{i}+a_{j}\right)} \mathbf{e}_{i j} \text { with } a=\frac{a_{i} a_{j}}{a_{i}+a_{j}}
$$

where $\mathbf{e}_{i j}$ is the normal vector towards the $j$ th particle

$$
\mathbf{e}_{i j}=\frac{\mathbf{X}_{i} \mathbf{X}_{j}}{\left\|\mathbf{X}_{i} \mathbf{X}_{j}\right\|}
$$




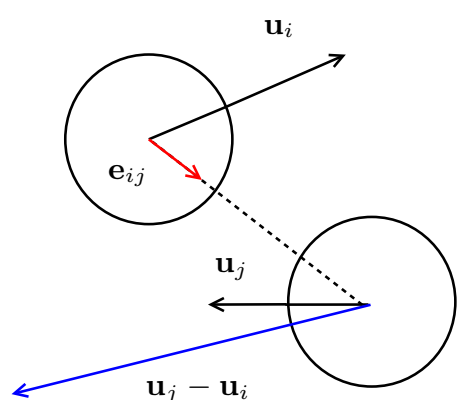

(a) Repulsion

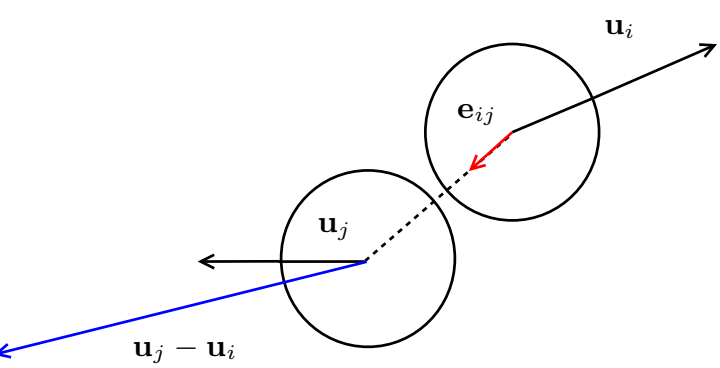

(b) Attraction

Figure 3. Effects of the lubrication force on the particle trajectories

As we can observe in expression [13], the lubrication force is acting in the same way of the term $\left(\mathbf{u}_{j}-\mathbf{u}_{i}\right) \cdot \mathbf{e}_{i j}$, as schematically explained in Figure 3 . Two different situations can typically occur:

- when $\left(\mathbf{u}_{j}-\mathbf{u}_{i}\right) \cdot \mathbf{e}_{i j}<0$, the particles are going closer to each other, and the lubrication force acts like a repulsion force,

- when $\left(\mathbf{u}_{j}-\mathbf{u}_{i}\right) \cdot \mathbf{e}_{i j}>0$, the particles are going away to each other, and the lubrication force acts like an attraction force.

With our approach, that means that the lubrication force will be able to avoid the overlap that can occur between the particles by adding a repulsive term in our numerical scheme.

Instead of adding directly this force into the weak formulation of the fluid-solid problem, the gluey model works like a predictor-corrector scheme. Indeed, adding the term corresponding to the hydrodynamic force would lead to a non-linearity that will be computationally more complicated and expensive to solve. The philosophy of the gluey model can also be summarized as follows:

1) in a first step, the fluid velocity is computed using the weak formulation [8],

2) and in a second step, the velocity would be corrected by taking into account more accurately the effects of lubrication forces in order to prevent the overlapping between particles.

\subsubsection{Correction of the predicted velocity}

The velocity field $\mathbf{u}^{*}$ obtained by solving Equation [8] does not take into account of contacts, that is why it is called predicted velocity. The gap between particles is defined by

$$
D_{i j}=\left\|\mathbf{X}_{i} \mathbf{X}_{j}\right\|-\left(a_{i}+a_{j}\right)
$$


and has to be positive. Indeed, in Equation [8], we do not have any information about a non-overlapping condition. This is precisely the first idea of the gluey model: we have to indicate which conditions must satisfy the velocity field in order to avoid overlapping. That is to say $D_{i j}^{n+1} \geq 0$ and a first order estimation of $D_{i j}^{n+1}$ reads

$$
D_{i j}^{n}+\Delta t\left(\mathbf{u}_{j}^{n}-\mathbf{u}_{i}^{n}\right) \cdot \mathbf{e}_{i j}^{n} \geq 0
$$

if the particle positions are updated by explicit scheme [11]. This is achieved by the following condition on the velocity field $\mathbf{u}^{n}$ :

$$
\frac{1}{2}\left|\mathbf{u}^{n}-\mathbf{u}^{*}\right|^{2}=\min _{\mathbf{v} \in K\left(\mathbf{X}^{n}, \gamma_{i j}^{n}\right)} \frac{1}{2}\left|\mathbf{v}-\mathbf{u}^{*}\right|^{2}
$$

where $K$ is the space of admissible velocity defined by

$$
K\left(\mathbf{X}^{n}, \gamma_{i j}^{n}\right)=\left\{\begin{array}{l|l}
\mathbf{v} & \begin{array}{l}
D_{i j}^{n}+\Delta t\left(\mathbf{v}_{j}-\mathbf{v}_{i}\right) \cdot \mathbf{e}_{i j}^{n} \geq 0 \text { if } \gamma_{i j}^{n}=0 \\
D_{i j}^{n}+\Delta t\left(\mathbf{v}_{j}-\mathbf{v}_{i}\right) \cdot \mathbf{e}_{i j}^{n} \leq 0 \text { if } \gamma_{i j}^{n}<0
\end{array}
\end{array}\right\}
$$

In these above constraints, $\gamma_{i j}^{n}$ is a new variable which represents the contact between particles $i$ and $j$ as follows:

$$
\gamma_{i j}^{n}\left\{\begin{array}{l}
<0 \text { if there is contact between particles } i \text { and } j \\
=0 \text { else }
\end{array}\right.
$$

Let us define the functional $J$ as follows:

$$
J(\mathbf{v})=\frac{1}{2}\left|\mathbf{v}-\mathbf{u}^{*}\right|^{2}=\frac{1}{2} \mathbf{M v} \cdot \mathbf{v}-\mathbf{M u}^{*} \cdot \mathbf{v}
$$

where $\mathbf{M}=\operatorname{diag}\left(\ldots, m_{i}, \cdots, m_{j}, \cdots\right)$ is the mass matrix. Then the Lagrangian of $J(\mathbf{u})$ for two particles $i$ and $j$ which takes into account of contact constraints has the following form (omitting the superscript $n$ for legibility):

$$
\begin{aligned}
\mathcal{L}\left(\mathbf{v}, \lambda_{i j}^{ \pm}\right)= & J(\mathbf{v})-\lambda_{i j}^{+}\left(D_{i j}+\Delta t\left(\mathbf{v}_{j}-\mathbf{v}_{i}\right) \cdot \mathbf{e}_{i j}\right) \\
& -\lambda_{i j}^{-}\left(-D_{i j}-\Delta t\left(\mathbf{v}_{j}-\mathbf{v}_{i}\right) \cdot \mathbf{e}_{i j}\right)
\end{aligned}
$$

where $\lambda_{i j}^{+}$and $\lambda_{i j}^{-}$are Lagrange multipliers. Finally, by solving $\frac{\partial \mathcal{L}}{\partial \mathbf{v}_{i}}=0$ and $\frac{\partial \mathcal{L}}{\partial \mathbf{v}_{j}}=0$, we obtain the two following relations:

$$
\left\{\begin{array}{l}
m_{i} \mathbf{u}_{i}^{n}=m_{i} \mathbf{u}_{i}^{*}-\left(\lambda_{i j}^{+}-\lambda_{i j}^{-}\right) \Delta t \mathbf{e}_{i j} \\
m_{j} \mathbf{u}_{j}^{n}=m_{j} \mathbf{u}_{j}^{*}+\left(\lambda_{i j}^{+}-\lambda_{i j}^{-}\right) \Delta t \mathbf{e}_{i j}
\end{array}\right.
$$

under the conditions :

$$
\lambda_{i j}^{ \pm}\left(D_{i j}+\Delta t\left(\mathbf{u}_{j}^{n}-\mathbf{u}_{i}^{n}\right) \cdot \mathbf{e}_{i j}\right)=0 \text { with } \lambda_{i j}^{+} \geq 0 \text { and } \lambda_{i j}^{-} \leq 0
$$

This minimization problem is also solved with an Uzawa procedure because this algorithm always converges even if there is no uniqueness of Lagrange multipliers for large numbers of contact. 


\subsubsection{Characterisation of the viscous contact}

In this section, we explain how we deal to characterise the viscous contact, namely how the variable $\gamma_{i j}$ is computed. In previous papers (Maury, 2007; Lefebvre, 2009), for 3D spherical particles driven by external forces (like gravity), $\gamma_{i j}$ is defined as the limit value of

$$
\gamma_{\eta, i j}=6 \pi \eta \ln \left(D_{i j}\right)
$$

as the the viscosity tends to zero. In fact $\gamma_{\eta, i j}$ describes the distance at microscopic level whereas $D_{i j}$ is the macroscopic distance between particles $i$ and $j$. That means that if $D_{i j}$ is below a specific value which depends on time step, mesh size or accuracy of numerical method, the two particles are assumed to be stuck. It is shown that the duration of this contact depends weakly on the viscosity and the evolution of the limit value $\gamma_{i j}$ is sufficient to get the total contact time (Lefebvre, 2007; Lefebvre, 2009). The relation between Lagrange multipliers and $\gamma_{i j}$ is achieved by using the Fundamental Principle of Dynamics with the lubrication forces for spherical particles:

$$
m_{i} \frac{d \mathbf{u}_{i}}{d t}=-a^{2} \frac{d \gamma_{i j}}{d t} \mathbf{e}_{i j}+m_{i} \mathbf{g}
$$

In their papers, $\mathbf{u}^{*}$ is the solution of the above equation without short range lubrication force and some basic calculus lead to the following relationship:

$$
\frac{d \gamma_{i j}}{d t}=-\frac{1}{a^{2}} \lambda_{i j} \quad \text { with } \quad \lambda_{i j}=\lambda_{i j}^{+}-\lambda_{i j}^{-}
$$

For solid particles in shear flow, the gravity and the inertia are usually neglected if the particles and fluid have same density. However by taking first order approximation of hydrodynamic force and assuming that $\mathbf{u}^{*}$ is solution of the Stokes equation for which the lubrication force is not taken into account carefully, one can gets the same relation [24] for $\gamma_{i j}=\ln \left(D_{i j}\right)$ and $\mathbf{M}=\Delta t \operatorname{diag}\left(\ldots, a_{i}, \cdots, a_{j}, \cdots\right)$ (Verdon $e t$ al., 2010). Moreover the reversibility of the Stokes equations implies that the time for which the Lagrange multiplier $\lambda_{i j}$ is positive ( that means that particle $i$ tends to go inside particle $j$ ) is the same that the time for which $\lambda_{i j}$ is negative ( particle $i$ tends to go outside particle $j$ ). Therefore the contact function $\gamma_{i j}$ can be connected to Lagrange multiplier by a simple relationship

$$
\frac{d \gamma_{i j}}{d t}=-\lambda_{i j}
$$

The case of inelastic collision is recovered by imposing a null contact function at any time and therefore the Lagrangian multiplier will be only positive.

\subsection{Final algorithm of the numerical procedure}

In this section we finally sum up the whole numerical procedure, including the gluey particle model. 


\section{1) Initialization}

$n=0$ (index for time step) : initialize $\mathbf{X}_{i}, \mathbb{I}$ and $\gamma_{i j}$ for all contacts

\section{2) Flow solver}

The weak formulation of the problem is solved with an augmented Lagrangian method and an Uzawa algorithm:

a) The velocity field $\mathbf{u}$ is computed by using a stable/stabilized finite element method for solving the weak formulation of the problem at step $k$ of the Uzawa procedure:

$$
\begin{aligned}
\int_{\Omega} 2 \eta \dot{\epsilon}\left(\mathbf{u}^{k}\right): \dot{\epsilon}(\mathbf{v}) d \Omega-\int_{\Omega} p^{k} \nabla \cdot \mathbf{v} d \Omega & =-\int_{\Omega} \mathbb{I} \eta_{s} \dot{\epsilon}\left(\lambda^{k-1}\right): \dot{\epsilon}(\mathbf{v}) d \Omega \\
\int_{\Omega} q \nabla \cdot \mathbf{u}^{k} d \Omega & =
\end{aligned}
$$

b) update of Lagrangian multiplier

$$
\lambda^{k}=\lambda^{k-1}+\mathbf{u}^{k}
$$

c) at convergence, we have the predicted velocity $\mathbf{u}^{*}$.

\section{3) Correction of velocity for an Adam-Bashforth scheme}

The predicted velocity $\mathbf{u}^{*}$ is corrected by the following Uzawa algorithm

a) let $\mathbf{w}^{0}$ be defined $\mathbf{w}^{0}=\mathbf{u}^{*}$ and choose arbitrarily $\delta_{i j}^{0}$ and $\rho>0$.

b) For all $k>0$, we compute $\mathbf{u}^{k+1}$ and $\delta_{i j}^{k+1}$ as follows:

$$
\begin{aligned}
& \mathbf{w}^{k+1}=\mathbf{u}^{*}+\frac{3 \Delta t}{2} \mathbf{M}^{-1} \mathbf{B}^{t} \delta_{i j}^{k} \\
& \quad \text { if } \gamma_{i j}^{n}<0, \quad \delta_{i j}^{k+1}=\delta_{i j}^{k}-\rho\left(\mathcal{D}_{i j}^{n}+\frac{3 \Delta t}{2} \mathbf{B} \mathbf{w}^{k+1}\right) \\
& \quad \text { if } \gamma_{i j}^{n} \geq 0, \quad \delta_{i j}^{k+1}=\max \left(0, \delta_{i j}^{k}-\rho\left(\mathcal{D}_{i j}^{n}+\frac{3 \Delta t}{2} \mathbf{B} \mathbf{w}^{k+1}\right)\right)
\end{aligned}
$$

where $\mathbf{B}=\left(-\mathbf{e}_{i j}^{n} \mathbf{e}_{i j}^{n}\right), \mathbf{M}=\operatorname{diag}\left(a_{i}, a_{j}\right)$ and

$$
\mathcal{D}_{i j}^{n}=D_{i j}^{n}-\frac{\Delta t}{2}\left(\mathbf{u}_{j}^{n-1}-\mathbf{u}_{i}^{n-1}\right) \cdot \mathbf{e}_{i j}^{n}
$$

c) at convergence, we have corrected velocity $\mathbf{u}^{n}$ and Lagrange multiplier $\lambda_{i j}^{n}$

\section{4) Update of contact functions $\gamma_{i j}$}

\section{For gluey contact}

$\gamma_{i j}^{n+1}=\gamma_{i j}^{n}-\Delta t \lambda_{i j}^{n}$

If $\gamma_{i j}^{n+1}<0$ then we have contact between particles $i$ and $j$ at next time step.

If $\gamma_{i j}^{n+1} \geq 0$, there is no more contact and $\gamma_{i j}^{n+1}=0$. 
For inelastic collision : $\gamma_{i j}^{n+1}=0$.

\section{5) Update of particle position}

it is done thanks to an Adams-Bashforth scheme (Hwang et al., 2004):

$$
\mathbf{X}_{i}\left(t^{n+1}\right)=\mathbf{X}_{i}\left(t^{n}\right)+\frac{\Delta t}{2}\left(3 \mathbf{u}^{n}-\mathbf{u}^{n-1}\right)
$$

\section{Results}

In this part we present the numerical improvements obtained by using the gluey particle model. As mentioned before, the main problem of previous results concerned the non-reversibility of the Stokes equations due to bad description of contact. That induces either an overlapping between particles, or a non-physical change in the trajectories coming from correction forces.

As illustrated in Figure 4, the problematic is checked on the evolution of particles in a shear flow. The shear flow is obtained by imposed velocities on the upper and lower walls (the shear rate is equal to 1 in all computations).

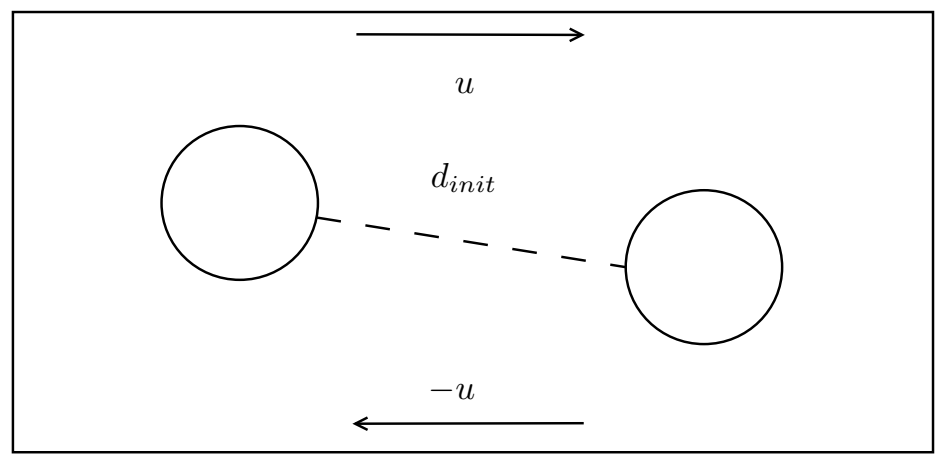

Figure 4. Two particles in a shear flow

In the following, we present results obtained with this configuration for both $2 \mathrm{D}$ and $3 \mathrm{D}$ flows. The computations are made with particles of radius $a=.05$ in an unitary box. It should be noted that particles must be seen rather as infinite cylinders for $2 \mathrm{D}$ computations. The last paragraph is devoted to examples of multi-particle computations.

\subsection{Computations for two particles in $2 D$ shear flow}

As emphasized in previous works, the minimal distance between particles in 2D shear flow remains larger than for 3D configuration (Meunier et al., 2008; Verdon et 
al., 2010). However, for a very small initial distance between two particles, the two particles rotate together and the minimal distance remains below $a \times 10^{-3}$. Therefore for large time step $\Delta t$, an overlapping can occur. For this purpose, we choose the following set of initial coordinates:

$$
\mathbf{X}_{1}=\left(\begin{array}{c}
0.451 \\
0.51
\end{array}\right) \text { and } \mathbf{X}_{2}=\left(\begin{array}{c}
0.549 \\
0.49
\end{array}\right)
$$

which corresponds to $D_{\text {init }}=2 \cdot 10^{-5}$. With these initial values, the different approaches can be checked:

- without correction, that is to say that there is no correction on velocity to prevent the overlapping between particles,

- with inelastic collision model, that is to say that $\gamma_{i j}=0$ at each time step,

- with gluey model, that is to say that $\dot{\gamma}_{i j}=-\lambda_{i j}$ as explained in the previous section.

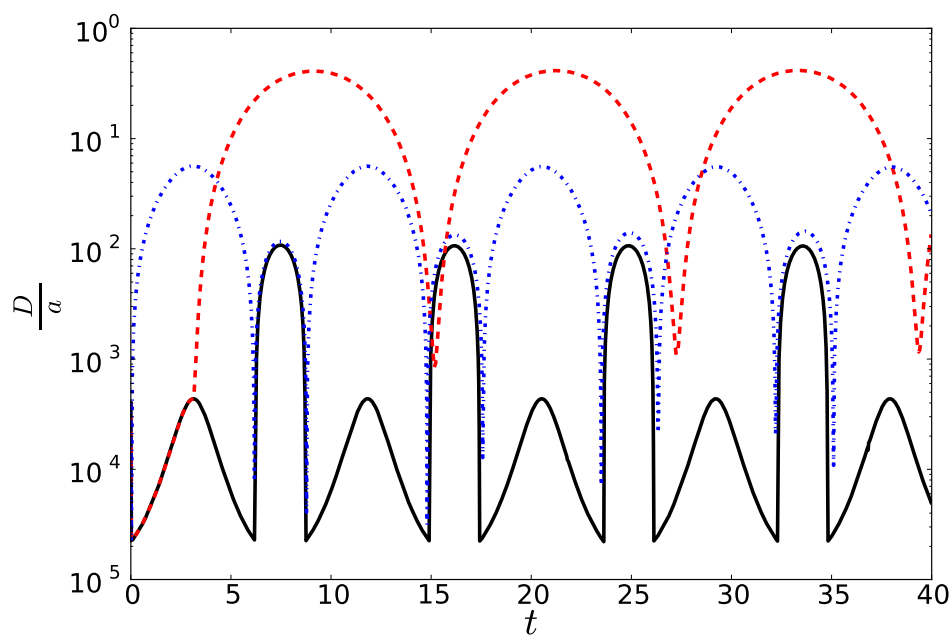

Figure 5. Two particles in $2 D$ shear flow: evolution of normalized distances $|D / a|$ between particles for the three different strategies (namely gluey contact (-), inelastic collision (- -) and without velocity correction (- .). For this latter case, the negative distance is also plotted

The computations are carried out by using a time step equal to $\Delta t=0.025$, and the results are summarized in Figures 5 and 6. From these figures we can make some important observations:

- the curve corresponding to the computation without correction (- .) is discontinuous because the absolute value of the negative distance is plotted (logarithmic scale). 


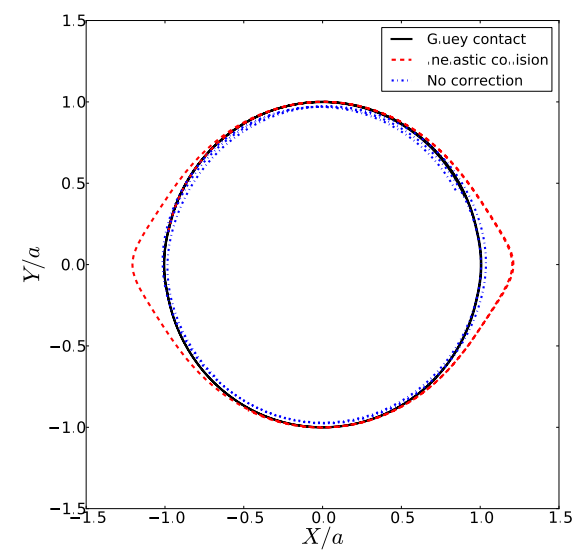

(a) Trajectories

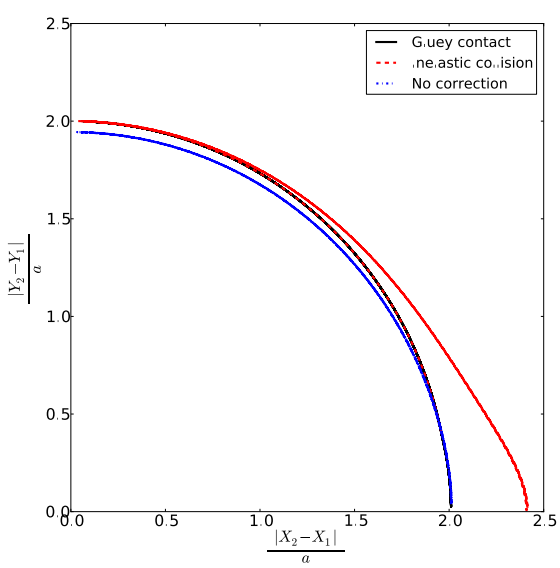

(b) Relative trajectories

Figure 6. Two particles in 2D shear flow: trajectories for gluey contact (-), inelastic collision (- -) and without velocity correction (- .)

When the distance is positive, the distance is the same as that given by the gluey model. When the distance is negative, the overlapping is rather important (bigger than $a \times 10^{-2}$ ) but thanks to Adam-Bashforth scheme, the particles do not remain sticked,

- with the gluey model, we recover the theoretical limit trajectory for a zero initial distance between two particles, namely a circle. When the gluey procedure is active, the distance between particle can be bigger than $a \times 10^{-2}$ because the constraint cancels only the first order expansion of $D^{n+1}$ and the term of order $\Delta t^{2}$ gives this positive distance. These value will decrease with the time step $\Delta t$,

- without correction, the trajectories of particles are similar to a circle, but with small fluctuations because of discrepancy due to overlapping,

- with the inelastic model, the particles change their trajectories, so they jump to a new orbit that is more ellipsoidal.

The results obtained by numerical scheme using gluey particle model indicate that circular trajectories are preserved, but at the same time the particles do not overlap. Hence we recover the theoretical trajectories without overlapping. 


\subsection{Two particles in a $3 D$ shear flow}

For spherical particles in a 3D shear flow, we choose a case for which the minimal distance between is very small. Two spheres with the same radius are placed in the superior and inferior mid-plane and move toward the right and the left side respectively. The time step is equal to $\Delta t=0.05$ and the initial positions are

$$
\mathbf{X}_{1}=\left(\begin{array}{c}
0.3 \\
0.525 \\
0.5
\end{array}\right) \text { and } \mathbf{X}_{2}=\left(\begin{array}{c}
0.7 \\
0.475 \\
0.5
\end{array}\right)
$$

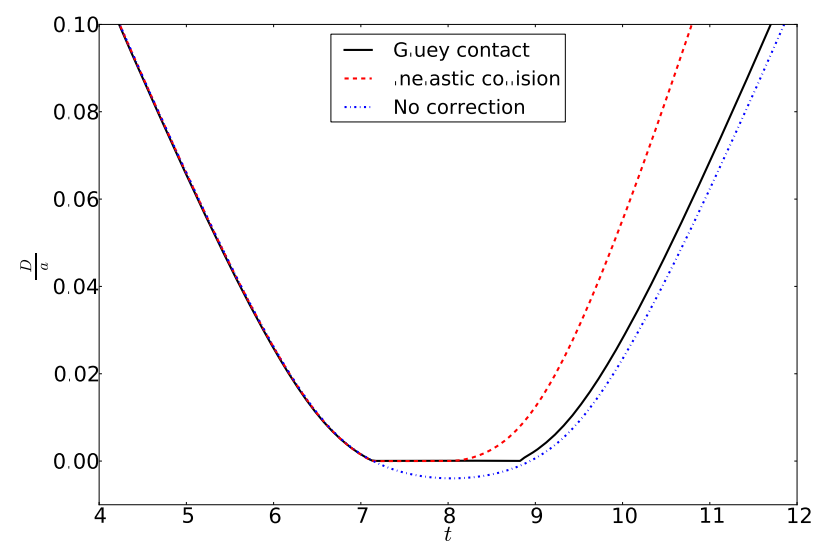

(a) Normalised distance between particles

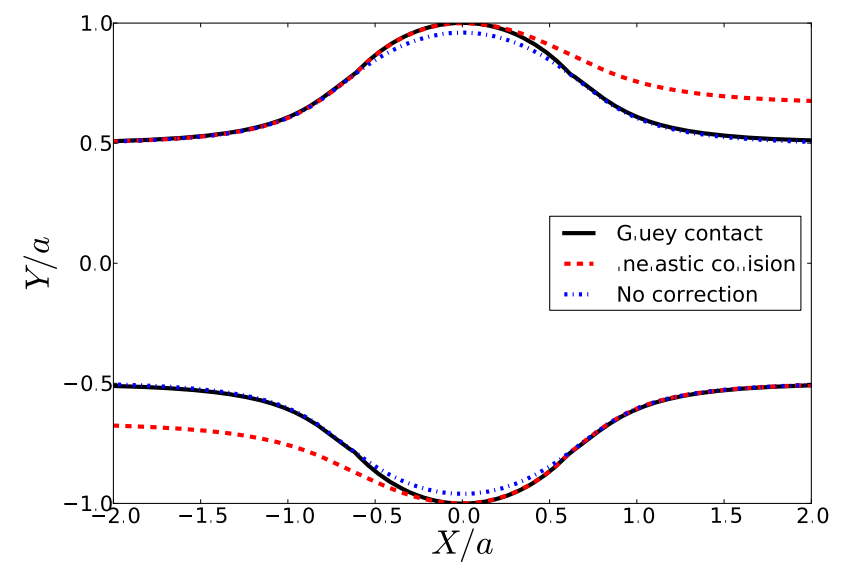

(b) Particle trajectories

Figure 7. Two particles in a 3D shear flow: comparison of collision strategies (gluey contact (-), inelastic collision (- -) and without velocity correction (- .) 
The Figures 7 show results obtained with the three different strategies already described. Once again, we observe a large overlapping if there is no correction on velocity field. The inelastic model avoids the overlapping but modifies significantly the trajectories: the reversibility is no more satisfied (it is both seen on the trajectory and the distance between the particles). With gluey contact, the reversibility is almost recovered but without overlapping (a better accuracy can be obtained with smaller time step). In addition, the relative error between the computed distance and the theoretical one when reversibility occurs is (at the end of the simulation) equals to $6.5 \%$ which is quite fair for 3D computations.

\section{3. $2 D$ multi-particle computations}

In this last paragraph, we will present results of multi-particle computations. The extension of algorithm described in previous section to multi-particle case can be found in (Lefebvre, 2009).

We present the motion of three particles in a shear flow. The main aim of this example is to check the influence of non-overlapping strategies on particle motions. The first two particles are located in the same position as in the example described in section 3.1 whereas the third particle is located away from the two others, namely at

$$
\mathbf{X}_{3}=\left(\begin{array}{c}
0.7 \\
0.49
\end{array}\right)
$$

as shown in Figure 8.

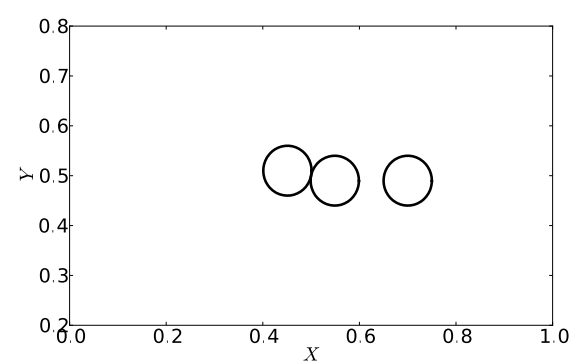

Figure 8. Initial position of three particles in $2 D$ shear flow

The computations were carried out until $t=30$ with a time step equal to $\Delta t=$ 0.025. In Figure 9, the relative distance between particles $D_{i j}$ are plotted for the three different strategies. The evolution of $D_{12}$ shows that the two first particles remain together for gluey model and if no correction is applied. On the other hand, they are separated with the inelastic collisions. Moreover without velocity correction, the third particle is also aggregated to the two other particles at time $t=30$ as the distance $D_{23}$ becomes smaller than $a \times 10^{-2}$. 


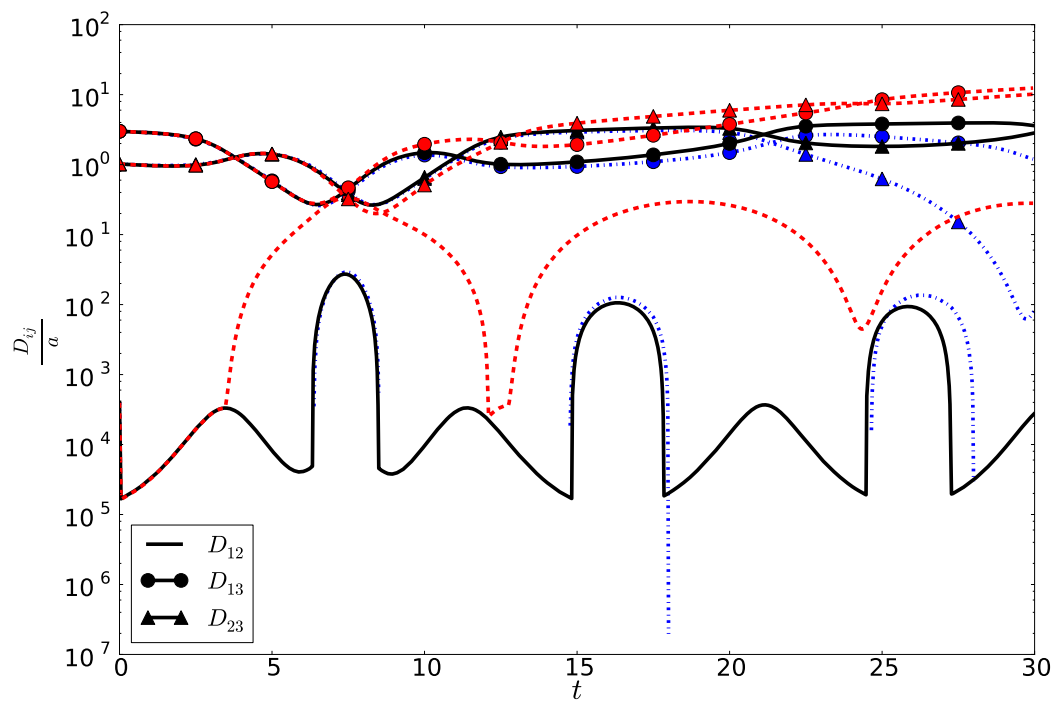

Figure 9. Normalised distances $D_{i j} /$ a between the three particles for gluey contact $(-)$, inelastic collision (- -) and without velocity correction (-.); in the latter case only positive distances are plotted

The time evolution of particle positions is plotted in Figures 10 and 11. From these figures we can make once again some important remarks:

- the results obtained with the three approaches are quite different, in particular with inelastic collision the three particles are no more close together,

- without correction of velocity field, there are overlapping between particles and therefore the three particles remain together at the end of computation time,

- the trajectories without velocity corrections and for gluey contacts are not the same because of the overlapping. The difference is bigger than in the case with two particles,

- due to the third particle, the flow motion is not symmetric with respect to the mid-plane. Therefore, the differences between trajectories obtained with these three approaches are more important.

Once again, the gluey model is the only model which avoids overlapping and prevents artificial numerical migration of particles in a suspension. These numerical illustrations proves that this algorithm is robust and well-suited for computations of multi-particle interactions in a fluid flow. 


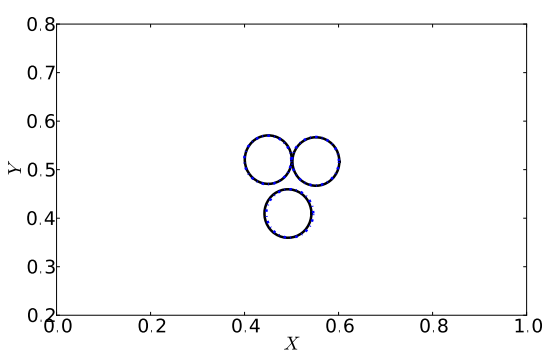

(a) $t=7.5$

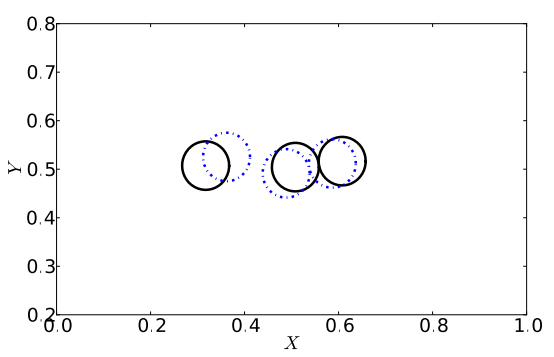

(c) $t=25$

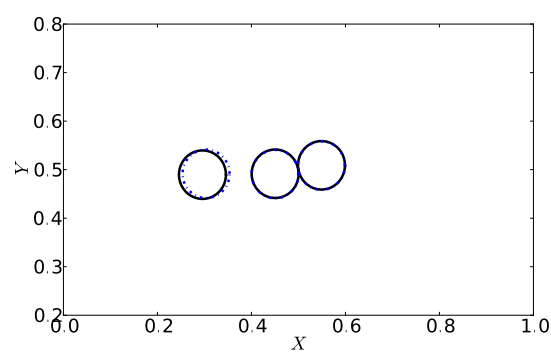

(b) $t=15$

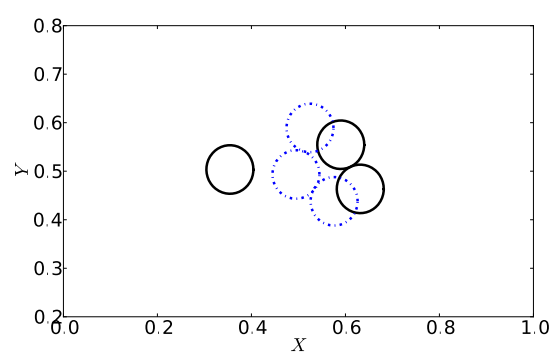

(d) $t=30$

Figure 10. Evolution of three particles in a 2D shear flow: comparisons between computations with gluey contact (-) and without velocity correction (- .)

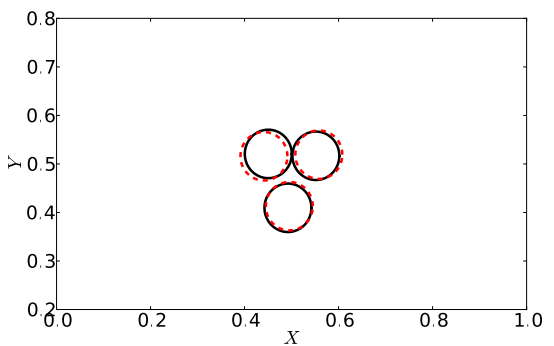

(a) $t=7.5$

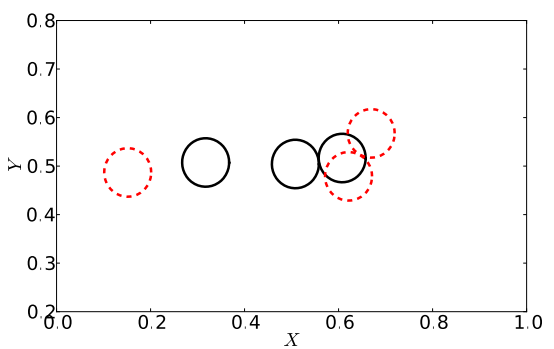

(c) $t=25$

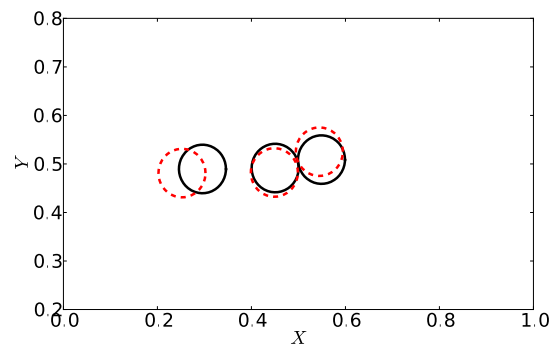

(b) $t=15$

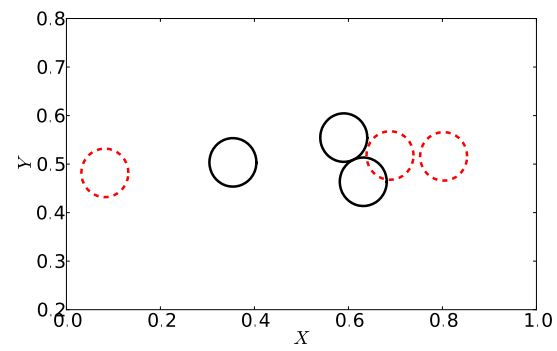

(d) $t=30$

Figure 11. Evolution of three particles in a $2 D$ shear flow: comparisons between computations with gluey contact (-) and with inelastic collision (- -) 


\section{Conclusion}

This article presents the adaptation of gluey particle model to study particle motions of in a shear flow. The gluey model has shown an improvement, especially by avoiding the classic overlapping between particles that can occur during the Lagrangian displacement of the particles. The immersed domain method associated to this contact model is then really effective to recover the reversibility of the Stokes equations. This result is particularly important for $3 \mathrm{D}$ computations because there are a lot of overlapping and artificial formation of aggregates. With the gluey model, we are able to avoid overlapping whereas the reversibility is preserved with a rather small relative error.

Furthermore, multi-particle examples have also been studied. In particular, the motion of three particles in a shear flow has been intensively studied. This example shows that the overlapping can be avoided by using the gluey model and that the resulting trajectories are really model-dependant. Therefore the choice of contact model has to be crucial for dense suspension computations.

Hence, future works will be devoted to the macroscopic impact of these microscopic modifications in the case of dense suspensions.

\section{Acknowledgements}

Computer time was provided by the CEMEF and the Mesocentre SIGAMM machine hosted by Observatoire de la Cote d'Azur. N. Verdon has received a grant from the ANR project RSC.

\section{References}

Coupez T., Digonnet H., Hachem E., Laure P., Silva L., Valette R., “ Multidomain Finite Element Computations: Application to Multiphasic Problems", in M. Souli, D. Benson (eds), Arbitrary Lagrangian-Eulerian and Fluid-Structure Interaction, Wiley, p. 229-297, 2009.

Fortin M., Glowinski R., Augmented Lagragian Methods, North-Holland, Amsterdam, 1983.

Glowinski R., Pan T.-W., Helsa T., Joseph D., “ A distributed Lagrange multiplier/fictious domain method for particulate flows", Int. J. Multiphase Flows, vol. 25, p. 755-794, 1999.

Glowinski R., Pan T.-W., Hesla T., Joseph D., Periaux J., “ A fictitious domain approach to the direct numerical simulation of incompressible viscous flow past moving rigid bodies : Application to particulate flow", J. Comput. Phys., vol. 169, p. 363, 2001.

Hwang W., Hulsen M., Meijer H. E. H., “ Direct simulations of particle suspensions in a viscoelastic fluid in sliding bi-periodic frames", J. Non-Newt. Fluid Mech., vol. 121, p. 15-33, 2004.

Laure P., Beaume G., Basset O., Silva L., Coupez T., “ Numerical methods for solid particles in particulate flow simulations”, European J. Comp. Mechanics, vol. 16, p. 365-383, 2007.

Lefebvre A., Modélisation numérique d'écoulements fluide/particules, $\mathrm{PhD}$ thesis, Université Paris Sud - Paris XI, 11, 2007. 
Lefebvre A., " Numerical simulation of gluey particles”, M2AN, vol. 43, p. 53-80, 2009.

Maury B., “A gluey particle model”, ESAIM: Proc., vol. 18, p. 133-142, 2007.

Meunier A., Bossis G., " The influence of surface forces on shear-induced tracer diffusion in mono and bidisperse suspensions", Eur. Phys. J. E, vol. 25, p. 187-199, 2008.

Patankar N., Singh P., Joseph D., Glowinski R., Pan T.-W., “ A new formulation of the distributed Lagrange multiplier/fictitious domain method for particulate flows", Int. J. of Multiphase flow, vol. 26, p. 1509-1524, 2000.

Singh P., Joseph D., " Sedimentation of a sphere near a vertical wall in an Oldroyd-B fluid", $J$. Non-Newtonian Fluid Mech., vol. 94, p. 179-203, 2000.

Verdon N., Beaume G., Lefebvre-Lepot A., Lobry L., Laure P., “ Immersed finite element method for direct numerical simulation of particle suspension in a shear flow", J. Comp. Physics, 2010.

Wachs A., “ A DEM-DLM/FD method for direct numerical simulation of particulate flows: Sedimentation of polygonal isometric particles in a Newtonian fluid with collisions", Computers \& Fluids, vol. 38, p. 1608-1628, 2009. 
